\title{
Magnetic Resonance for Differential Diagnosis of Left Ventric- ular Hypertrophy: diagnostic and prognostic implications
}

\author{
Giovanni Donato Aquaro, 1,*, Elisabetta Corsi 2, Giancarlo Todiere 1, Crysanthos Grigoratos 1, Andrea Barison 1, \\ Valerio Barra ${ }^{1}$, Gianluca Di Bella ${ }^{3}$, Michele Emdin 1,4, Fabrizio Ricci, Alessandro Pingitore 5.
}

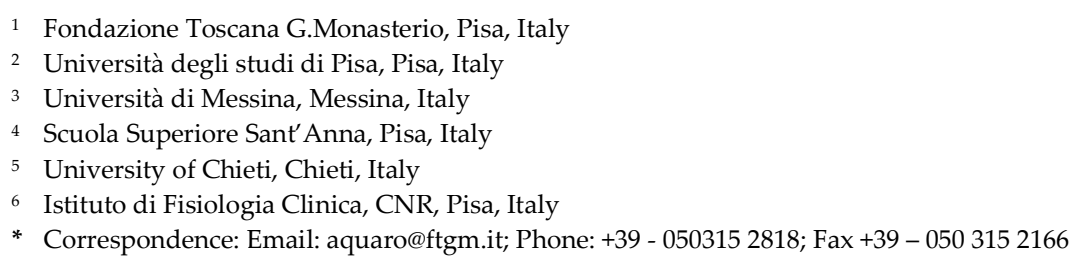

\begin{abstract}
Background: Left ventricular hypertrophy (LVH) may be due to different causes, ranging from benign secondary forms to severe cardiomyopathies. Transthoracic Echocardiography (TTE) and ECG are the first level examination for LVH diagnosis. Cardiac magnetic resonance (CMR) defines accurately LVH type, extent and severity. Objectives: to evaluate the diagnostic and prognostic role of CMR in patients with TTE and/or ECG evidence of LVH. Methods: We performed CMR in 300 consecutive patients with echocardiographic and/or ECG signs of LVH.Results: Overall, 275 patients had TTE evidence of LVH with initial suspicion of hypertrophic cardiomyopathy (HCM) in $132(44 \%)$, cardiac amyloidosis in 41 (14\%), hypertensive LVH in $48(16 \%)$, aortic stenosis in $4(1 \%)$, undetermined LVH in $50(16 \%)$. The initial echocardiographic diagnostic suspicion of LVH was confirmed in 172 patients $(57.3 \%)$ and changed in 128 patients $(42.7 \%$, $\mathrm{p}<0.0001$ ): the diagnosis of $\mathrm{HCM}$ increased from $44 \%$ to $71 \%$ of patients; hypertensive and undetermined LVH decreased significantly (respectively to $4 \%$ and $5 \%$ ). CMR allowed a diagnosis in 41 out of $50(82 \%)$ with undetermined LVH at TTE. CMR also identified HCM in 17 out of 25 patients with apparently normal echo but with ECG criteria for LVH. Finally, the reclassification of the diagnosis by CMR was associated with a change of survival risk of patients: after CMR reclassification no events occurred in patients with undetermined or hypertensive LVH. Conclusions: CMR changed echocardiographic suspicion in almost half of patients with LVH. In the subgroup of patient with abnormal ECG, CMR identified LVH (particularly HCM) in $80 \%$ of patients. This study highlights the indication of CMR to better characterize the type, extent and severity of LVH detected at echocardiography and suspected with ECG.
\end{abstract}

Keywords: Left ventricular hypertrophy; Echocardiography; Magnetic Resonance; Prognosis

\section{INTRODUCTION}

The presence of left ventricular hypertrophy (LVH) is a recognized independent risk factor for cardiac-related morbidity and mortality (1). However, LVH does not represent a unique disease entity but rather the phenotypic expression of a large spectrum of diseases, such as hypertrophic cardiomyopathy or amyloidosis, as well as a physiologic adaptation, as in the case of athlete's heart. Thus, the clinical and prognostic weight depends on the type of the disease causing LVH and also the extent and the severity of myocardial damage (2). This highlights the need to characterize as best as possible LVH in terms of diagnosis and severity. Currently, transthoracic echocardiography (TTE) represents the diagnostic entrance door of $\mathrm{LVH}$, providing with high accuracy to measure LV wall thickness and to stratify patients for the risk of sudden death on the basis of the entity of wall 
thickness (3). In addition, also LV outflow tract obstruction (LVOT) and left atrial enlargement are included in the risk score assessment of sudden death (4). Cardiac magnetic resonance (CMR) has higher potential to define wall thickness and the extent of LVH in comparison to TTE due to higher spatial resolution and multiplanar approach that makes it a three dimensional imaging technique. This is in particular evident in the apical region that is barely valuable with TTE $(5,6)$. In addition, CMR provides information regarding myocardial tissue characterization with the identification of fibrosis and edema (7-9), that have potential relevance in the risk stratification of patients with LVH (10). In particular, late gadolinium enhancement (LGE) provides the opportunity to define accurately the diagnostic nature of $\mathrm{LVH}$ according to the intramyocardial distribution and the wash out curve of the gadolinium (11). The majority of the studies comparing CMR and TTE for the LVH diagnosis is mainly focused on HCM (12-15). Actually, there are no studies showing the additive value of CMR over TTE in the definition of LVH of different nature. Therefore, the aim of the present study was to assess the diagnostic impact of CMR in comparison to TTE for the detection of LVH and the diagnostic definition on the nature of LVH. Moreover, we also assessed the prognostic impact of CMR in patients with LVH diagnosis as defined with this technique.

\section{METHODS}

We performed CMR in 300 consecutive patients with the suspicion of LVH, defined as the presence of at least one of the following criteria: 1) LVH detected by echocardiography; 2) ECG criteria for LVH (ECG voltage criteria for $\mathrm{LVH}, 16)$. The following inclusion criteria were further used: 12-leads resting ECG and TTE; age > 18 years; absence of contraindication for CMR; known history of cardiac disease and/or systemic disease potentially involving heart; LV ejection fraction $>45 \%$.

\subsection{LVH at echocardiography}

Standard echocardiographic examinations of any patients was performed following the current ESC guidelines (17). LV hypertrophy during TTE was defined as maximal diastolic wall thickness $\geq 11 \mathrm{~mm}$ with a two dimensional (2D) echocardiography-guided Mmode approach in the parasternal long axis. (14) The initial echocardiographic diagnostic suspicion was performed as follows. Briefly, HCM was suspected in presence of asymmetric LVH with maximal end diastolic wall thickness $\geq 15 \mathrm{~mm}$ with or without LVOT obstruction (defined as an instantaneous peak Doppler LV outflow tract pressure gradient $\geq 30 \mathrm{mmHg}$ at rest or during physiological provocation such as Valsalva manoeuvre, standing and exercise). In patients with concentric hypertrophy (maximal end diastolic wall thickness $\geq 15 \mathrm{~mm}$ ), the presence of LVOT functional obstruction was used as suspected HCM (3).

Cardiac amyloidosis was suspected in presence of concentric hypertrophy, ground glass appearance of ventricular myocardium on 2D echocardiography, diastolic dysfunction, thickening of interatrial septum, global LV hypokinesia with or without apical sparring (3).

Hypertensive LVH was defined for patients with systemic arterial hypertension (systolic blood pressure $\geq 140 \mathrm{mmHg}$ or diastolic blood pressure $\geq 90 \mathrm{mmHg}$ during office measurements), LVH with maximal end diastolic wall thickness $\leq 14 \mathrm{~mm}$, in absence of any other secondary cause of LVH.(18).

In patients with LVH, the presence of moderate-severe aortic stenosis was considered as the main cause of hypertrophy in absence of any other causes. Patients were assigned to aortic stenosis group in the presence of moderate ( $\mathrm{V} \max \leq 4 \mathrm{~m} / \mathrm{s}, \Delta \mathrm{Pm} \leq 40 \mathrm{mmHg}$, AVA $>1 \mathrm{cmq}$ ) or severe (V max $\geq 4 \mathrm{~m} / \mathrm{s}, \Delta \mathrm{Pm} \geq 40 \mathrm{mmHg}) \cdot(19)$

Athlete's heart was defined in patients practicing intense sport training $\geq 2$ hours per day for at least 5 days per week in the last 12 months having eccentric hypertrophy, LV dilation, preserved systolic and diastolic function with normal regional wall motion.(20). 
Finally, undetermined LVH was defined in case of absence of any of the above mentioned criteria.

\subsection{Cardiac Magnetic Resonance evaluation}

The examination was performed using 1.5 Tesla scanner (Signa Hdx, General Electric Healthcare, Milwaukee, Wisconsin) with dedicated cardiac phased array coil. Study protocol included functional evaluation with short axis cine images, acquired from the mitral plane valve to the left ventricular (LV) apex, and these images were acquired using a steady-state free precession pulse sequence with the following parameters: 30 phases, slice thickness $8 \mathrm{~mm}$, no gap, views per segment 8 , FOV $35-40 \mathrm{~cm}$, phase FOV 1, matrix $224 \times 224$, reconstruction matrix $256 \times 256,45^{\circ}$ flip angle, and a TR/TE near to 2 . Cine images with the same parameters were acquired also in 2-,3- and 4 chamber views.

According to the protocols recommended by the Society for Cardiovascular Magnetic Resonance, we acquired T1 mapping sequence using a MOdified Look-Locker Inversion Recovery (MOLLI) method with 3(3)3(3)5 protocol. A 3(3)3(3)5 scheme indicates that there are a total of 3 inversions; 3 images are acquired after the first inversion, this is followed by a waiting period of 3 RR intervals, then 3 images are acquired followed by another 3 RR waiting period, finally a third inversion after which 5 images are acquired, for a total of 11 images over 17 heartbeats.

We obtained three parallel short-axis slices, including the base, mid-cavity, and apex of the left ventricle, at the same cardiac phase (end diastole).

LGE images were acquired 10 minutes after the administration of Gd-DTPA with a dosage of $0.2 \mathrm{mmol} / \mathrm{kg}$ in short-axis views. An inversion recovery T1-weighted gradientecho (GRE) sequence was used with the following parameters: field of view $35-40 \mathrm{~mm}$, slice thickness $8 \mathrm{~mm}$, no gap between each slice, repetition time 3-5 msec, echo time 1-3, a flip angle of $25^{\circ}$, matrix $224 \times 224$, reconstruction matrix $256 \times 256$. The appropriate inversion time was set to null for normal myocardium using a TI-scout.

All CMR studies were analysed offline in the core laboratory of Pisa by three experienced observers with III level EACVI accreditation for CMR investigators who were blinded to the clinical data. A commercially available research software package (Mass Analysis, Leyden, The Netherlands) was used to quantify the functional parameters using conventional method. Left ventricular mass was measured by the analysis of the cine short-axis images. The endocardial and epicardial contours of LV myocardium were manually traced in the end-diastolic and the end-systolic phases. End-diastolic volume index, end-systolic volume index, mass, and mass index were measured as previously described $(21,22)$. Maximal LV end diastolic wall thickness was measured as previously described. The pattern of LVH was defined as asymmetric (septal, septo-apical, apical, inferolateral), concentric and eccentric. The maximal end diastolic wall thickness was measured. The pattern of LGE was measured and classified as follows: ischemic pattern (subendocardial/transmural, located in coronary artery territory), non-ischemic (midwall or subepicardial, non-respecting coronary artery territory) (11). The presence of the specific pattern of cardiac amyloidosis was also detected (diffuse subendocardial enhancement, early darkening of the cavity, nulling defect of myocardium) (23).

Native myocardial T1 was evaluated by the generation of T1 maps and the measurement of myocardial T1 performed for each myocardial segments.

Combining morphological, functional and tissue findings, the differential diagnosis of LVH was performed as follows. Briefly, HCM was diagnosed in patients with asymmetrical LVH with maximal diastolic wall thickness $\geq 15 \mathrm{~mm}$ (or an apex/base wall thickness ratio $>1$ for apical pattern), with or without LGE. In patients with concentric LVH, the presence of LVOT functional obstruction was considered as a proof of HCM. Cardiac amyloidosis was identified in patients with concentric LVH with the specific pattern of LGE. Hypertensive LVH was defined for patients with LVH with maximal end diastolic wall thickness $\leq 14 \mathrm{~mm}$, negative LGE and with the exclusion of any other secondary cause of LVH. In patients with LVH, the presence of moderate/severe aortic stenosis was 
considered as the main cause of hypertrophy in absence of LGE and any other causes. Athlete's heart was defined in patients practicing intense sport training $\geq 2$ hours per day for at least 5 days per week in the last 12 months having eccentric hypertrophy, balanced biventricular dilation (RV EDV/LV EDV in the range 0.85-1.15), preserved systolic function with normal regional wall motion and absence of LGE. Finally, undetermined LVH was defined in case of absence of any of the above mentioned criteria and absence of LGE.

\subsection{Additional evaluation}

In order to confirm the diagnosis after the echocardiographic and CMR suspicion, patients underwent to further evaluations based on clinical indication. For instance, in the suspicion of cardiomyopathy, genetic evaluation was performed for the identification of specific pathogen mutations, and in the suspect of cardiac amyloidosis, diphosphonate scintigraphy and umbilical fat biopsy was performed.

\subsection{Clinical follow-up}

After the CMR examination, a follow-up was performed. A clinical questionnaire was compiled by a clinical physician during periodic ambulatory visitations in each hospital, by contacting their relatives by telephone, or by a general practitioner. The clinical questionnaire included the definition of the following cardiac events: cardiac death, resuscitated cardiac arrest and appropriate implantable cardioverter defibrillator (ICD) shock, anti-tachycardia pacing, sustained ventricular tachycardia on Holter electrocardiogram monitoring, heart failure hospitalization. A complete analysis of the ICD was performed by the referring cardiologist in order to confirm the appropriateness of the shock. A panel of expert investigators adjudicated the occurrence of cardiac events.

\subsection{Statistical analysis}

Values are presented as the mean +- standard deviations (SD) or as the median (interquartile range IQR) for variables with normal and non-normal distributions, respectively. Values with non-normal distribution according to Kolmogorov-Smirnov test were logarithmically transformed for parametric analysis. Qualitative data are expressed as percentages. Categorical variables were compared by the chi-square test or the Fisher exact test when appropriate. Continuous variables were compared by the ANOVA t test and analysis of variance or by the Wilcoxon nonparametric test when appropriate. Bonferroni correction was used when needed. The Kaplan-Meier time-to-event method was used to calculate and compare longitudinal curves among groups. A p value lower than 0.05 was considered statistically significant.

\section{RESULTS}

Among the 300 enrolled patients: 275 (92\%) had LVH at echocardiography; 151(50\%) had ECG abnormalities (ECG criteria of LVH in 76, 25\%); 25 had ECG signs of LVH with negative TTE $(8 \%)$.

The characteristics of the population are summarized in Table 1. Table 2 shows TTE diagnostic suspicions, CMR diagnosis of LVH and the diagnostic discrepancies between the two techniques. In particular, after CMR, the initial TTE diagnostic suspicion of LVH was confirmed in 173 patients $(57.7 \%)$ and changed in 127 patients $(42.3 \%$, p<0.0001) (Figure 1) 


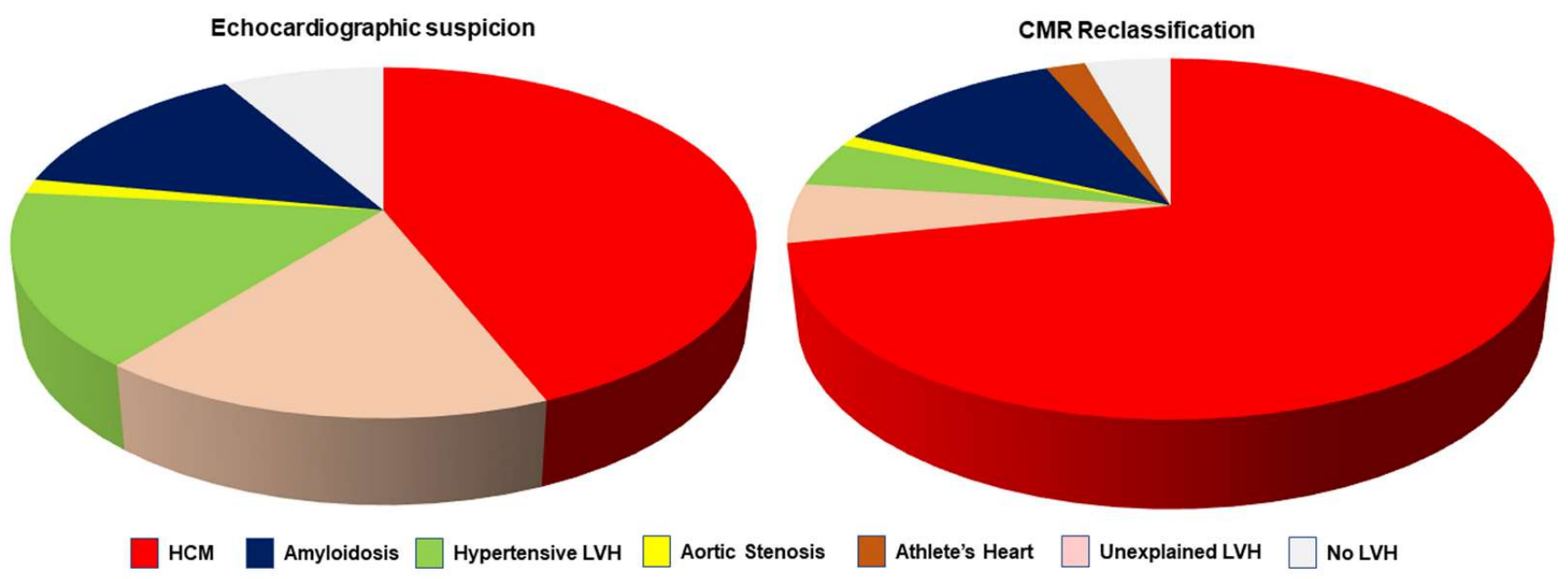

Figure 1. The left graph shows the distribution of initial diagnostic suspicion by transthoracic echocardiography (TTE). The right graph shows the reclassification of diagnosis by cardiac magnetic resonance (CMR). As evident by the comparison of the two graphs, CMR allowed a significant decrease of cases with undetermined left ventricular hypertrophy (LVH), as well a decrease of the percentage of hypertensive LVH. HCM, hypertrophic cardiomyopathy.

Table 1. Characteristics of the whole population ( $n=300$ patients).

\begin{tabular}{|c|c|}
\hline Age(year) & $56 \pm 18$ \\
\hline Males, n(\%) & $220(73 \%)$ \\
\hline Height $(\mathrm{cm})$ & $171 \pm 9$ \\
\hline Weight (Kg) & $76 \pm 14$ \\
\hline Hypertension, n (\%) & $119(40 \%)$ \\
\hline Dyslipidaemia, n (\%) & $64(21 \%)$ \\
\hline Diabetes, n (\%) & $29(10 \%)$ \\
\hline Familial history of CAD, n (\%) & $24(8 \%)$ \\
\hline Familial history of cardiomyopathy, n (\%) & $17(6 \%)$ \\
\hline Familial history of SCD, n (\%) & $23(8 \%)$ \\
\hline Atrial fibrillation, n (\%) & $26(9 \%)$ \\
\hline ECG abnormalities: & $151(50 \%)$ \\
\hline Sokolow LVH criteria, n (\%) & $76(25 \%)$ \\
\hline T negative, $\mathrm{n}(\%)$ & $89(20 \%)$ \\
\hline Left bundle branch block, $\mathrm{n}(\%)$ & $9(3 \%)$ \\
\hline Low voltages on peripheral leads, n(\%) & $4(1 \%)$ \\
\hline Short PR interval, n (\%) & $2(1 \%)$ \\
\hline Echographic criteria of LVH, n (\%) & $275(92 \%)$ \\
\hline \multicolumn{2}{|l|}{ LVH patterns at echo: } \\
\hline Septal, n (\%) & $131(44 \%)$ \\
\hline Concentric, n (\%) & $127(42 \%)$ \\
\hline Septo-apical, n (\%) & $2(0.7 \%)$ \\
\hline Apical, n (\%) & $7(2 \%)$ \\
\hline Infero-lateral, n (\%) & $1(0.3 \%)$ \\
\hline Maximal end-diastolic wall thickness at echo (mm) & $15(13-18)$ \\
\hline Diastolic dysfunction, n (\%) & $193(64 \%)$ \\
\hline Grade $1, \mathrm{n}(\%)$ & $127(42 \%)$ \\
\hline Grade $2, \mathrm{n}(\%)$ & $37(12 \%)$ \\
\hline Grade 3, n (\%) & $29(10 \%)$ \\
\hline LVOT obstruction, n (\%) & $31(10 \%)$ \\
\hline Systolic dysfunction, n (\%) & $35(12 \%)$ \\
\hline Aortic valvular stenosis, $\mathrm{n}(\%)$ & $4(1 \%)$ \\
\hline
\end{tabular}


CAD: coronary artery disease; SCD: sudden cardiac death; LVH: left ventricular hypertrophy; LVOT: left ventricular outflow tract.

Table 2. Comparison between echocardiographic suspicion and CMR final diagnosis in the different groups; in the grey boxes, the matches for each group, n (\%). LVH: left ventricular hypertrophy.

\begin{tabular}{|c|c|c|c|c|c|c|c|c|}
\hline & \multicolumn{8}{|c|}{ CMR Reclassification: } \\
\hline TTE suspicion: & HCM & CA & $\begin{array}{c}\text { Hypertensive } \\
\text { LVH }\end{array}$ & $\begin{array}{c}\text { Aortic } \\
\text { stenosis }\end{array}$ & $\begin{array}{c}\text { Undetermined } \\
\text { LVH }\end{array}$ & $\begin{array}{c}\text { Athlete's } \\
\text { heart }\end{array}$ & No LVH & Sum \\
\hline HCM & $124(94 \%)$ & 2 & 2 & 0 & 1 & 0 & 3 & $\begin{array}{c}132 \\
(44.0 \%)\end{array}$ \\
\hline CA & 9 & $23(56 \%)$ & 2 & 0 & 5 & 0 & 2 & $41(13.7 \%)$ \\
\hline $\begin{array}{c}\text { Hypertensive } \\
\text { LVH }\end{array}$ & 36 & 3 & $8(17 \%)$ & 0 & 0 & 0 & 1 & $48(16.0 \%)$ \\
\hline Aortic stenosis & 1 & 0 & 0 & $3(75 \%)$ & 0 & 0 & 0 & $4(1.4 \%)$ \\
\hline $\begin{array}{l}\text { Undetermined } \\
\text { LVH }\end{array}$ & 28 & 7 & 0 & 0 & $9(18 \%)$ & 4 & 2 & $50(16.7 \%)$ \\
\hline \multirow[t]{2}{*}{ No LVH } & 17 & 0 & 0 & 0 & 1 & 2 & $5(20 \%)$ & $25(8.3 \%)$ \\
\hline & $215(71.7 \%)$ & $\begin{array}{c}35 \\
(11.7 \%)\end{array}$ & $\begin{array}{c}12 \\
(4.0 \%)\end{array}$ & $\begin{array}{c}3 \\
(1.0 \%)\end{array}$ & $\begin{array}{c}16 \\
(5.0 \%)\end{array}$ & $\begin{array}{c}6 \\
(2 \%)\end{array}$ & $\begin{array}{c}13 \\
(4.3 \%)\end{array}$ & 300 \\
\hline
\end{tabular}

${ }^{*}$ Others diagnosis were made by CMR in 3 patients (1\%): 1 ischemic heart disease, 2 with no LVH but non ischemic LGE.

Among the 48 patients with TTE suspicion of hypertensive LVH, CMR excluded any other different diagnosis in 8 cases (17\%), whereas a CMR suggested a different diagnosis in 40: HCM in 36 cases (75\%); patients typical LGE features of cardiac amyloidosis in $3(6 \%)$; non-ischemic LGE without hypertrophy in 1 (example in figure 2). 


\section{SSFP}

\section{T1 mapping}

\section{LGE}
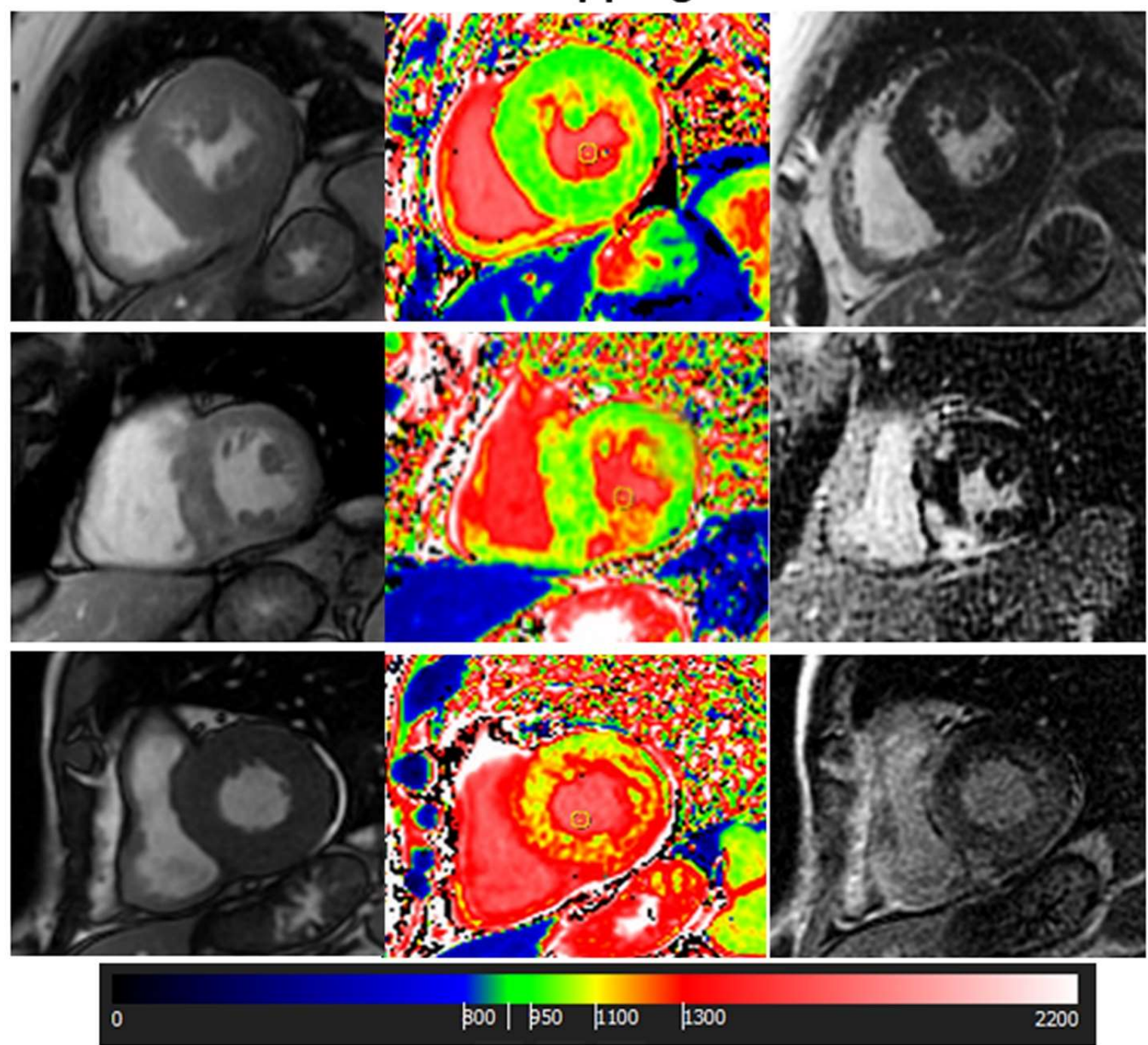

Figure 2. Cardiac Magnetic Resonance of 3 different cases of left ventricular hypertrophy: The upper panels show a case of hypertensive left ventricular hypertrophy (LVH) with concentric hypertrophy, normal native T1 values and negative late gadolinium enhancement (LGE). The middle panels show a case of Hypertrophic cardiomyopathy (HCM) with asymmetrical LVH, focal areas of myocardial fibrosis at LGE and focal increase of native T1 corresponding to the fibrotic areas. Finally, a case of cardiac amyloidosis is reported in the lower panels. In this case LVH is concentric, native T1 is diffusely increased and a diffuse subendocardial enhancement was found at LGE.

Among 132 patients with TTE suspicion of HCM, CMR confirmed this diagnosis in 124 out of 132 patients (94\%), while the remaining 8 patients were reclassified as follows: 2 patients with cardiac amyloidosis (1.5\%); 2 with hypertensive LVH $(1.5 \%)$, 1 case with concentric hypertrophy without LGE $(0.8 \%)$ having a short native myocardial T1 (with subsequent genetic diagnosis of Fabry disease, figure 3); in the last 3 cases hypertrophy was excluded and LGE was negative (2.2\%). 


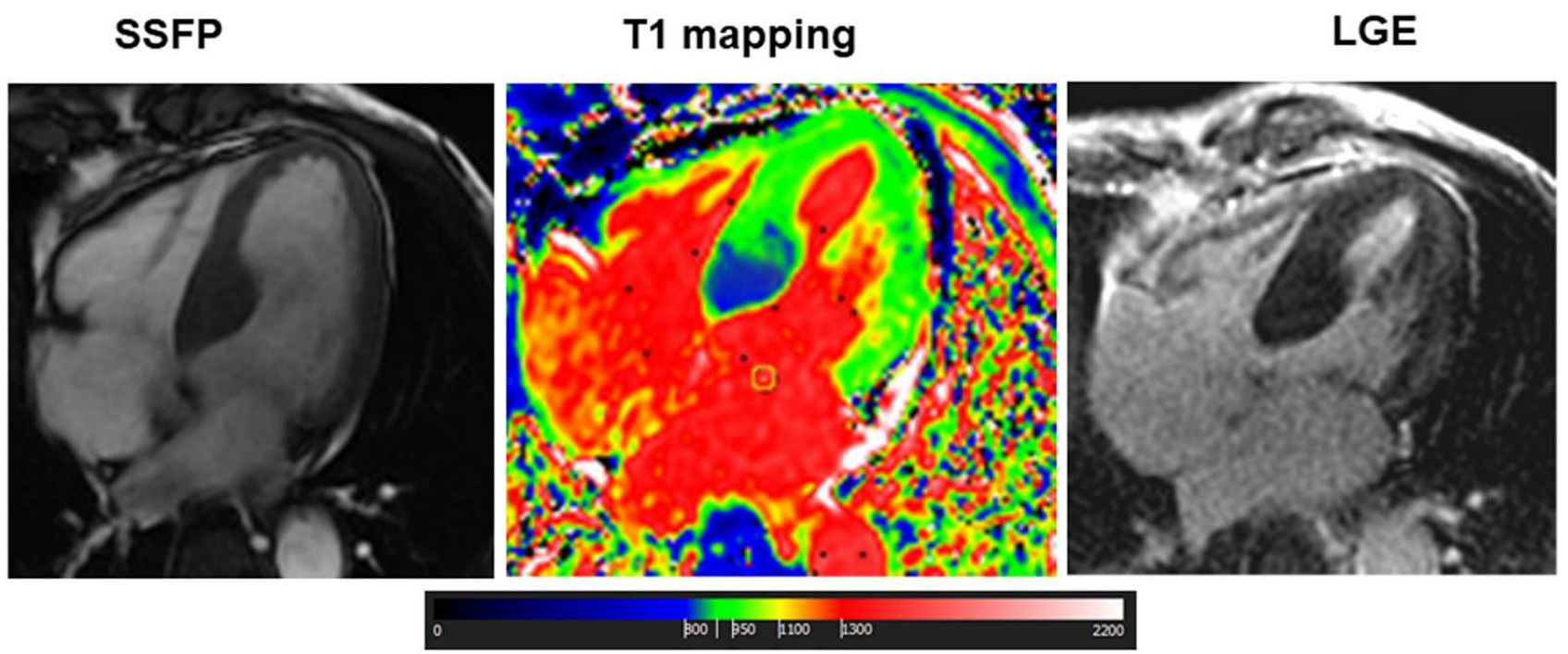

Figure 3. Cardiac Magnetic Resonance of a patient with Fabry disease: This was a case of a 70 years old male with apparently asymmetrical LVH, with extensive LGE in the lateral wall but with low myocardial T1 in the basal septum. This latter finding suggested the diagnosis of Fabry disease with pseudo-normalization of T1 in the fibrotic myocardial segment of lateral wall. The subsequent genetic evaluation confirmed the presence of a pathogenic mutation of alfa-galactosidases.

Among 41 patients with TTE suspicion of amyloidosis, CMR confirmed this diagnosis in 23 patients (56\%). Typical features of amyloidosis were absent in the remaining 19 cases: in 9 patients HCM was found (22\%), 2 patients $(5 \%)$ had hypertensive LVH, 2 patients $(5 \%)$ had athlete's heart, 1 pt $(2 \%)$ had ischemic LGE without hypertrophy suggesting previous myocardial infarction. Finally, in 4 patients (10\%) LV concentric hypertrophy with negative LGE was found.

Aortic stenosis was confirmed as cause of hypertrophy in 3 out of 4 patients (75\%). In the remaining patient HCM was diagnosed.

The diagnosis of athlete's heart of was confirmed in only one subject by CMR.

CMR permitted to make a diagnosis in 40 out of 49 patients $(81 \%)$ with undetermined hypertrophy at echo: HCM was diagnosed in 28 patients $(57 \%)$, athlete's heart in 2 patients (4\%), amyloidosis in 7 (14\%), whereas LVH was excluded in 3 patients (1\%).

Finally, among the 25 patients with ECG criteria of LVH but with negative TTE, 5 $(18 \%)$ had normal CMR. Seventeen of them $(68 \%)$ were HCM, 2 patients $(8 \%)$ had athlete's heart and in 1 patient (4\%) the cause of LVH remained undetermined. Characteristics of diagnostic groups are showed in table 3.

Table 3. Comparison of patient groups based on CMR reclassification

\begin{tabular}{|c|c|c|c|c|c|c|c|}
\hline & $\begin{array}{l}\text { HCM } \\
(n=215)\end{array}$ & $\begin{array}{c}\text { Amyloidosis } \\
(n=35)\end{array}$ & $\begin{array}{l}\text { Hypertensive LVH } \\
(n=12)\end{array}$ & $\begin{array}{l}\text { Athletes's heart } \\
(n=6)\end{array}$ & $\begin{array}{c}\text { Aortic stenosis } \\
(n=3)\end{array}$ & $\begin{array}{c}\text { Undetermined LVH } \\
(\mathrm{n}=16)\end{array}$ & p \\
\hline Age (years) & $56 \pm 16^{2,4}$ & $68 \pm 11^{1,4}$ & $60 \pm 14^{4}$ & $18 \pm 5^{*}$ & $73 \pm 17^{4}$ & $50 \pm 22^{4}$ & $<0.001$ \\
\hline Males, $\mathrm{n}(\%)$ & $156(73 \%)$ & $28(80 \%)$ & $9(75 \%)$ & $7(100 \%)$ & $2(67 \%)$ & $10(67 \%)$ & 0.37 \\
\hline Hypertension, $\mathrm{n}(\%)$ & $92(43 \%)^{2,3}$ & $6(17 \%)^{1,3}$ & $12(100 \%)^{1,2,4,6}$ & $2(29 \%)^{3}$ & $2(67 \%)$ & $0^{3}$ & 0.007 \\
\hline Diabetes, $\mathrm{n}(\%)$ & $27(13 \%)^{2}$ & $0^{1}$ & $1(8 \%)$ & 0 & 0 & 0 & 0.84 \\
\hline Dyslipidaemia, n (\%) & $56(26 \%)^{6}$ & $4(11 \%)$ & $3(25 \%)$ & 0 & $1(33 \%)$ & $0^{1}$ & 0.45 \\
\hline $\begin{array}{c}\text { Family history of CAD, } \\
\mathrm{n}(\%)\end{array}$ & $14(7 \%)$ & 0 & $1(8 \%)$ & 0 & 0 & $2(13 \%)$ & 0.04 \\
\hline ECG signs of LVH, n (\%) & $25(12 \%)^{2}$ & $0^{1,3}$ & $3(25 \%)^{2}$ & 0 & $1(33 \%)$ & $2(13 \%)$ & 0.29 \\
\hline T negative, $\mathrm{n}(\%)$ & $44(20 \%)$ & $4(11 \%)$ & $2(17 \%)$ & $1(14 \%)$ & $1(33 \%)$ & $2(13 \%)$ & 0.19 \\
\hline Low voltages, n (\%) & $0^{2}$ & $4(11 \%)^{1}$ & 0 & 0 & 0 & 0 & 0.016 \\
\hline $\begin{array}{l}\text { Diastolic dysfunction: } \\
\text { Grade 1, n (\%) }\end{array}$ & & & & & & $4(29 \%)$ & $<0.0001$ \\
\hline
\end{tabular}




\begin{tabular}{|c|c|c|c|c|c|c|c|}
\hline Grade 2, n (\%) & $108(50 \%)^{2,4}$ & $5(14 \%)^{1,3}$ & $8(67 \%)^{2,4}$ & 01,3 & $2(67 \%)$ & $2(14 \%)$ & \\
\hline Grade 3, n (\%) & $17(8 \%)^{2}$ & $16(46 \%)^{1,4}$ & $2(17 \%)$ & $0^{2}$ & 0 & $0^{2}$ & \\
\hline & $15(7 \%)^{2}$ & $14(40 \%)^{1,3,6}$ & $0^{2}$ & 0 & 0 & & \\
\hline \multicolumn{8}{|c|}{ CMR parameters } \\
\hline $\begin{array}{l}\text { Max telediastolic wall } \\
\text { thickness (mm) }\end{array}$ & $19 \pm 4$ & $19 \pm 4$ & $14 \pm 2$ & $13 \pm 2$ & $18 \pm 2$ & $15 \pm 3$ & 0.001 \\
\hline LVEF (\%) & $69 \pm 11^{2}$ & $54 \pm 4^{1,3,4,6}$ & $66 \pm 14^{2}$ & $70 \pm 8^{2}$ & $53 \pm 12$ & $68 \pm 9^{2}$ & $<0.001$ \\
\hline LVEDVi $(\mathrm{ml} / \mathrm{mq})$ & $73 \pm 19$ & $76 \pm 27$ & $77 \pm 23$ & $97 \pm 21$ & $87 \pm 27$ & $77 \pm 18$ & 0.14 \\
\hline Mass (g/mq) & $95 \pm 26$ & $115 \pm 36$ & $87 \pm 26$ & $104 \pm 18$ & $130 \pm 37$ & $96 \pm 28$ & $<0.001$ \\
\hline RVEF (\%) & $69 \pm 92$ & $53 \pm 14^{1}$ & $67 \pm 13$ & $66 \pm 7$ & $62 \pm 9$ & $65 \pm 11$ & $<0.001$ \\
\hline RVEDVi (ml/mq) & $67 \pm 17^{4}$ & $66 \pm 174$ & $71 \pm 17$ & $105 \pm 23^{1,2}$ & $62 \pm 10$ & $80 \pm 23$ & $<0.001$ \\
\hline Septal LVH, n (\%) & $155(72 \%)^{2,4,6}$ & $8(23 \%)^{1}$ & $6(50 \%)$ & $1(14 \%)^{1}$ & $1(33 \%)$ & $4(27 \%)^{1}$ & $<0.0001$ \\
\hline Septal apical LVH, n (\%) & $12(6 \%)$ & 0 & 0 & 0 & 0 & 0 & 0.83 \\
\hline Apical LVH, n (\%) & $18(8 \%)$ & 0 & 0 & 0 & 0 & 0 & 0.58 \\
\hline Concentric LVH, n (\%) & $28(13 \%)^{2,3,4,6}$ & $26(74 \%)^{1}$ & $6(50 \%)^{1}$ & $5(72 \%)^{1}$ & $2(67 \%)$ & $10(67 \%)^{1}$ & 0.88 \\
\hline $\begin{array}{l}\text { Inferior and inferolateral } \\
\qquad \mathrm{LVH}, \mathrm{n}(\%)\end{array}$ & $2(1 \%)$ & 0 & 0 & 0 & 0 & 0 & 0.98 \\
\hline Presence of LGE, n (\%) & $163(76 \%)^{2,3,4,6}$ & $34(97 \%)^{*}$ & $0^{1,2}$ & 01,2 & $1(33 \%)^{2}$ & $0^{1,2}$ & $<0.001$ \\
\hline Abnormal native T1 & $104(48 \%)$ & $35(100 \%)$ & 0 & 0 & $1(33 \%)$ & 0 & $<0.001$ \\
\hline \multicolumn{8}{|c|}{$\begin{array}{l}\text { A p value }<0.05 \text { was considered significant. }{ }^{1} \text { vs } \mathrm{HCM} ;{ }^{2} \text { vs amyloidosis; }{ }^{3} \text { vs hypertensive LVH; }{ }^{4} \\
\text { vs athlete's heart; }{ }^{5} \text { vs aortic stenosis; }{ }^{6} \text { vs undetermined LVH; }{ }^{*} \text { vs all other groups .CAD: coronary } \\
\text { artery disease; LVH: left ventricular hypertrophy; LVEF: left ventricle ejection fraction; LVEDVi: } \\
\text { left ventricle end diastolic volume indexed; RVEF: right ventricle ejection fraction; RVEDVi: right } \\
\text { ventricle end diastolic volume indexed }\end{array}$} \\
\hline
\end{tabular}

\subsection{Factors determining CMR vs TTE changes in LVH diagnosis}

Maximal wall thickness (MWT), LVH site, LGE pattern and native T1 mapping were considered as key element to change CMR diagnosis of LVH in respect to TTE. In general, the calculation of MWT between the 2 techniques significantly correlated $(\mathrm{r}=0.80$, $\mathrm{p}<0.001)$. However, average maximal thickness was higher in $\operatorname{CMR}(17,15-21)$ vs echo $(16$, 14-19 mm; median difference $1 \mathrm{~mm}$, range 1-3 mm, $\mathrm{p}<.0001)$. The higher difference was in the HCM group (median $2 \mathrm{~mm}$, range 1-4 mm, p<.001) and in the amyloidosis group (median $2 \mathrm{~mm}$, range 1-3 mm). In 65 patients, CMR detected a MWT higher than $15 \mathrm{~mm}$ whereas at TTE MWT was lower.

TTE identified 98 out of 155 (63\%) with septal hypertrophy at CMR, 15 out 30 (50\%) with apical hypertrophy, 11 of the 28 (39\%) with concentric hypertrophy and none of the 2 with inferior/inferolateral hypertrophy. TTE was more able to detect septal HCM than other patterns $(\mathrm{p}=0.008)$. LGE pattern was also useful for the final diagnosis. The typical LGE pattern of amyloidosis allowed to identify 34 patients $(97 \%)$, while in 1 patient a diffuse and severe increase of native T1, summed to concentric hypertrophy allowed the diagnosis. In HCM, LGE was found in the mid-wall layer of hypertrophic segments in 163 patients (76\%). LGE with ischemic pattern was found in 1 patient with LVH due to aortic stenosis. Finally, as mentioned above, in 1 patient with concentric hypertrophy and negative LGE, the finding of decrease native myocardial T1 suggested the diagnosis of Fabry disease that was confirmed through the genetic evaluation.

\subsection{Clinical follow-up}

Clinical follow-up was performed in 272 patients for a median of 2440 (937-3421) days, 45 major cardiac events occurred (20 cardiac deaths, 3 appropriate ICD intervention and 22 hospitalization for heart failure). The Kaplan Meier curves of figure 3, show the survival-free from events of patients with one of the major 4 diagnoses: HCM, cardiac amyloidosis, hypertensive LVH and undetermined LVH. As shown in the figure, the reclassification of diagnosis made by CMR was also associated with a change in the risk of events among the groups. Indeed, after the CMR reclassification 23 out of 45 events occurred in patients with cardiac amyloidosis and 22 in those with HCM, whereas no events occurred in hypertensive or undetermined LVH. 
Combined Endpoint based on Echocardiographic suspicion

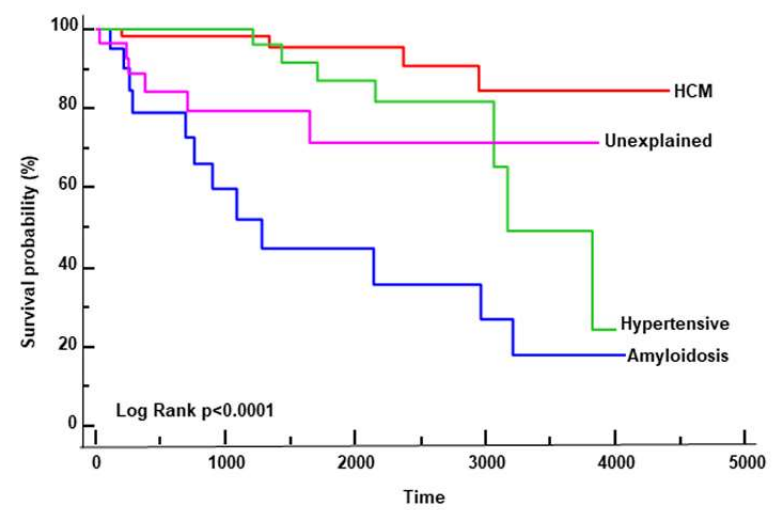

Number at risk

\begin{tabular}{|c|c|c|c|c|c|c|}
\hline HCM & 60 & 44 & 22 & 13 & 3 & 0 \\
\hline Amyloidosis 2 & 22 & 8 & 5 & 3 & 2 & 0 \\
\hline Hypertensive 3 & & 26 & 17 & 7 & 1 & 0 \\
\hline Unexplained 2 & & 16 & 9 & 4 & 0 & 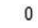 \\
\hline
\end{tabular}

Combined Endpoint based on CMR reclassification

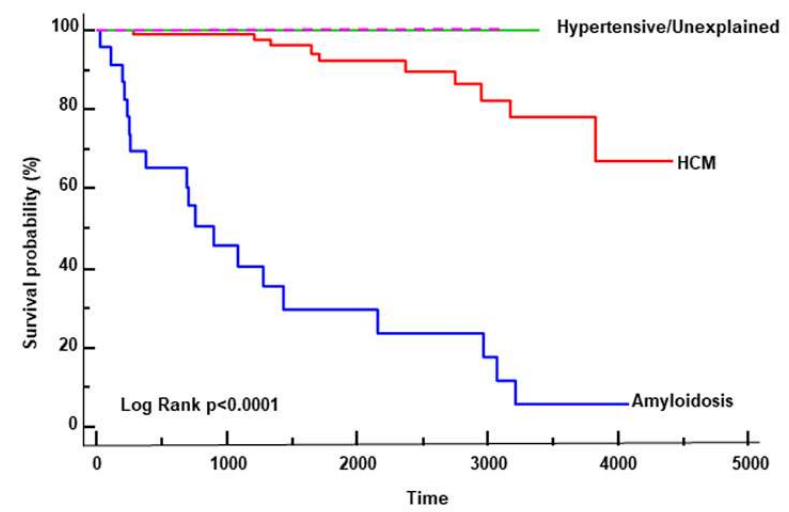

Number at risk:

$\begin{array}{lllllll}\text { HCM } & 112 & 78 & 40 & 20 & 4 & 0 \\ \text { Amyloidosis } & 23 & 9 & 5 & 3 & 1 & 0 \\ \text { Hypertensive } & 10 & 4 & 4 & 3 & 0 & 0 \\ \text { Unexplained } & 5 & 1 & 1 & 0 & 0 & 0\end{array}$

Figure 4. Kaplan-Meier curves: The graphs represent the survival-free from events curve of the patients with the 4 major diagnosis in this population: hypertrophic cardiomyopathy (HCM), cardiac amyloidosis, hypertensive left ventricular hypertrophy $(\mathrm{LVH})$ and undetermined LVH. The left graph shows the curves based on the initial echocardiographic suspicion, the right graph was the result of the reclassification made by cardiac magnetic resonance (CMR). As evident, the reclassification allowed a prognostic change in survival curves: no events occurred in patients with hypertensive LVH and those with undetermined LVH based on CMR diagnosis. .

\section{DISCUSSION}

The main results of this study can be summarized in the following points:

1. In patients with LVH at echo or with ECG signs of hypertrophic phenotype, CMR changed the initial echocardiographic suspicion in $42.3 \%$ cases, in particular changing the diagnosis of hypertensive $\mathrm{LVH}$ in $43 \%$ cases

2. The factors changing diagnosis were MWT measurement, the pattern of LVH, with average MWT higher in CMR vs echo. Tissue characterization, with LGE and T1 mapping, was also effective to identify HCM and cardiac amyloidosis.

3. The final diagnosis by CMR was prognostically relevant providing an accurate patient risk stratification, in line with the type of diagnosis.

In general, this study showed that CMR allows a more accurate differentiation among the phenotypes of hypertrophy than echocardiography. This was due mainly to higher precision in the measurement of MWT, the identification of hypertrophic segments that are unlikely visualized with echocardiography, and tissue characterization by the assessment of the presence and the pattern of myocardial fibrosis or amyloid material, defined according to LGE technique and by the T1 mapping technique. Previous studies showed that TTE can both underestimate or overestimate LV wall thickness, the first one due to poor acoustic window or focal hypertrophy, the second one due to LV trabeculation, right ventricular myocardium, papillary muscle, imaging plane obliquity apical septal bundle (13-15). However, in the study of Bois JB et al, CMR identified higher number of patients with massive LV hypertrophy than TTE, confirming a discrepancy between these 2 techniques (13). Interestingly, a recent study showed that the prevalence of unexplained LV hypertrophy in a population based cohort was $1.4 \%$ with CMR (24) Whether these discrepancies were mainly documented in the setting of patients with HCM, in this study, we documented that the discrepancies involve also other types of LVH. Indeed, 
CMR changed diagnosis of the type of LVH in $42 \%$ of patients. In particular, this occurred in the group of TTE-diagnosed hypertensive LVH, in which CMR confirmed diagnosis only in $17 \%$ of patients, and changed diagnosis mainly in the type of HCM. The identification of asymmetrical LVH with MWT >15 mm (underestimated or undetected by TTE), summed, in many cases, by the detection of mid-wall LGE in hypertrophied segments, were the major reasons for the change of diagnosis from hypertensive LVH to HCM.

Also, CMR changed TTE-diagnosis of amyloidosis in a large number of patients, that is $56 \%$ of them. Among these, $22 \%$ of patients had CMR-diagnosis of HCM, and the others had variegated type of LVH. In this setting of patients, changes in diagnosis was due to the assessment of LGE pattern, that provides accurate information on the presence of amyloid material, in which the most common pattern is a diffuse or sub-endocardial distribution of LGE with a non-coronary artery distribution, early darkening of the blood pool, and defect of myocardial nulling (25). T1 mapping played also an important role in the evaluation of cardiac amyloidosis, showing a diffuse and severe increase of myocardial native T1(whereas T1 increased patchy in HCM, decreased in Fabry disease and was normal in hypertensive LVH). T1 mapping was also crucial for the diagnosis of the patient with Fabry disease: the finding of a decreased myocardial T1, summed to the inferolateral midwall LGE, allowed to suspect this diagnosis (figure 4)

Also, CMR provided certain diagnosis in patients with undetermined diagnosis at TTE; in this group the main CMR diagnosis was HCM, with an average difference between MWT at CMR and at TTE of $3.8 \pm 2 \mathrm{~mm}$.

Differently, CMR confirmed TTE-diagnosis of HCM in $94 \%$ of patients. Among these, 40 had LVOT obstruction. It is noteworthy that $76 \%$ of these patients had intramyocardial LGE. Several studies have already shown that the presence and the extent of LGE is associated to wall thickness, and is an independent predictor of severe ventricular arrhythmias and sudden death $(10,26)$. Further, fibrosis is a progressive phenomenon over the years and its increment is associated to a worse clinical status, highlighting the need of a CMR longitudinal follow up (27).

The effectiveness of CMR in the diagnosis of the different types of LVH is expressed in terms of prognosis. Indeed, event did not occur in patients with a final CMR diagnosis of hypertensive LVH and in those who remained with undetermined LVH diagnosis. This reclassification of the risk of cardiac events made by $\mathrm{CMR}$ is consistent with the available data about the risk of cardiac events in cardiomyopathy as amyloidosis and HCM compared to hypertensive LVH $(28,29)$. An important point is that prognostic risk stratification profile due to CMR diagnostic changes in LVH patterns, suggests that TTE risk stratification profile worsened, in particular in TTE groups of hypertensive LVH due to the erroneous presence of other more prognostically severe LVH forms. Accordingly, in this study, we showed that in the TTE group of LVH hypertension, patients with HCM or amyloidosis were also included. Therefore, it is reasonable to consider again the prognostic weight of TTE LVH in hypertensive patients. Thus, the clinical impact of these data is that CMR may be useful to define the prognostic risk of LVH.

\section{Limitations of the study}

This is a real life study and it was performed without the use of more advanced echocardiographic techniques as speckle tracking, 3D-echo. These more complex echocardiographic techniques could have probably improved the accuracy of the echocardiographic diagnosis, mainly in the cases of uncertain diagnosis.

\section{CONCLUSIONS}

In this study, we showed that CMR and TTE have discrepancies in the diagnostic definition of the type of $\mathrm{LVH}$, depending by several factors that include the identification of LVH of myocardial segments poorly visualized by TTE, the maximal wall thickness, the availability of a more accurate analysis of myocardial tissue characterization with 
LGE. Thus, this study asks the question whether CMR may be more frequently used in patients with TTE evidence of LVH with the goal of a more accurate diagnostic and prognostic definition. Further studies are needed to answer this query.

\section{Clinical perspective}

Clinical competency in medical knowledge: Cardiac magnetic resonance enhance (CMR) our ability to identify the causes of left ventricular hypertrophy compared to transthoracic echocardiography. After CMR the initial diagnostic suspicion changes in $>40 \%$ of cases and this is associated with a substantial variation of the risk of cardiac events of the patients. This study highligths that CMR should be performed in every patients with echocardiographic evidence of left ventricular hypertrophy.

Translational outlook: The reclassification of the risk of cardiac events of patients with left ventricular hypertrophy consequent to CMR diagnosis, suggests the need to reevalute the data of the prognostic risk associated with some cardiac conditions, obtained in pre-CMR era.

\section{REFERENCES}

1. Levy D, Garrison RJ, Savage DD, Kannel WB, Castelli WP. Prognostic implications of echocardiographically determined left ventricular mass in the Framingham Heart Study. N Engl J Med 1990;322:1561-1566

2. Devereux RB, Roman MJ. Left ventricular hypertrophy in hypertension: stimuli, patterns, and consequences. Hypertens Res 1999;22:1-9

3. Authors/Task Force members, Elliott PM, Anastasakis A, Borger MA et al. 2014 ESC Guidelines on diagnosis and management of hypertrophic cardiomyopathy: the Task Force for the Diagnosis and Management of Hypertrophic Cardiomyopathy of the European Society of Cardiology (ESC). Eur Heart J 2014;35:2733-2779

4. O'Mahony C. A novel clinical risk prediction model for sudden cardiac death in hypertrophic cardiomyopathy (HCM riskSCD). Eur Heart J 2014;35:2010-2020

5. Moon JC, Fisher NG, McKenna WJ, Pennell DJ. Detection of apical hypertrophic cardiomyopathy by cardiovascular magnetic resonance in patients with non-diagnostic echocardiography. Heart. 2004;90:645-649

6. Bottini PB, Carr AA, Prisant LM, Flickinger FW, Allison JD, Gottdiener JS. Magnetic resonance imaging compared to echocardiography to assess left ventricular mass in the hypertensive patient. Am J Hypert 1995;8:221-228

7. Aquaro GD, Positano V, Pingitore A et al. Quantitative analysis of late gadolinium enhancement in hypertrophic cardiomiopathy. J Cardiovasc Magn Reson 2010; 7:12-21

8. Burrage MK, Ferreira VM. Cardiovascular magnetic resonance for the differentiation of left ventricualr hypertrophy. Current Heart Failure Reports 2020;17:192-204

9. Karamitsos TD, Arvanitaki A, Karvounis H, Neubaer S, Ferreira VM. Myocardial tissue characterization and fibrosis by imaging. JACC Cardiovascular Imaging. 2020;13:1221-1234

10. Chan RH, Maron BJ, Olivotto I et al. Prognostic value of quantitative contrast-enhanced cardiovascular magnetic resonance for the evaluation of sudden death risk in patients with hypertrophic cardiomyopathy. Circulation. 2014;130:484:495

11. Mahrholdt H, Wagner A, Judd RM, Sechtem U, Kim RJ. Delayed enhancement cardiovascular magnetic resonance assessment of non-ischaemic cardiomyopathies, Eur Heart J 2005;26:1461-1474

12. Rickers C, Wilke NM et al. Utility of cardiac magnetic resonance imaging in the diagnosis of hypertrophic cardiomyopathy. Circulation 2005;112:855-861

13. Bois JP, Geske JB, Foley TA, Ommen SR, Pellikka PA. Comparison of maximal wall thickness in hypertrophic cardiomyopathy differs between magnetic resonance imaging and transthoracic echocardiography. Am J Cardiol 2017; 119:643-650

14. Devlin AM, Moore NR, Ostman-Smith I. A comparison of MRI and echocardiography in hypertrophic cardiomyopathy. Br J Radiol 1999;72:258-264

15. Hindieh W, Weissler-Snir A, Hammer H, Adler A, Rakowsku H, Chan RH. Discrepant measurements of maximal left ventricular wall thickness beteen cardiac magnetic resonance imaging and echocardiography in patients with hypertrophic cardiomyopathy. Circ Cardiovasc Imaging 2017;10:e006309

16. Sokolow M, Lyon TP. The ventricular complex in left ventricular hypertrophy as obtained by unipolar and limb leads. Am Heart J 1949;37:161-86

17. Lang, Badano LP, Mor-Avi V et al. Recommendations for Cardiac Chamber Quantification by Echocardiography in Adults: An Update from the American Society of Echocardiography and the European Association of Cardiovascular Imaging, J Am Soc Echocardiogr 2015;28:1-39.

18. Williams B, Mancia G, Spiering W, et al. 2018 ESC/ESH Guidelines for the management of arterial hypertension. Eur Heart J 2018;39:3021-3104

19. Baumgartner H, Falk V, Bax JJ et al. ESC Scientific Document Group, 2017 ESC/EACTS Guidelines for the management of valvular heart disease. Eur Heart J 2017;38:2739-2791 
20. Pelliccia A, Maron MS, Maron BJ, Assessment of Left Ventricular Hypertrophy in a Trained Athlete: Differential Diagnosis of Physiologic Athlete's Heart From Pathologic Hypertrophy. Prog Cardiovasc Dis 2012;54:387-96

21. Aquaro GD, Camastra G, Monti L et al. Working group "Applicazioni della Risonanza Magnetica" of the Italian Society of Cardiology.Reference values of cardiac volumes, dimensions, and new functional parameters by MR: A multicenter, multivendor study. J Magn Reson Imaging. 2017;45:1055-1067.

22. Kramer CM, Barkhausen J, Bucciarelli-Ducci C, Flamm SD, Kim RJ, Nagel E. Standardized cardiovascular magnetic resonance imaging (CMR) protocols: 2020 update. J Cardiovasc Magn Reson. 2020;22:17

23. Fontana, M., Chung, R., Hawkins, P.N. et al. Cardiovascular magnetic resonance for amyloidosis. Heart Fail Rev 2015;20: 133

24. Massera D, Mcclelland RL, Ambale-Venkatesh B et al. Prevalence of unexplained left ventricular hypertrophy by cardiac magnetic resonance imaging in MESA. J Am Heart Assoc 2019;8:e012250

25. Syed IS, Glockner JF, Feng DL et al. Role of Cardiac Magnetic Resonance Imaging in the Detection of Cardiac Amyloidosis. JACC: Cardiovascular Imaging 2010;3:155-164

26. Weng Z, Yao J, Chan RH, He J, Yang X, Zhou Y, He Y, Prognosis value of LGE-CMR in HCM: a meta-analysis. JACC Cardiovasc Imaging 2016;9:1392-1402

27. Todiere G, Aquaro GD, Piaggi P et al. Progression of myocardial fobrosis assessed with cardiac magnetic resonance in hypertrophic cardiomyopathy. J Am Coll Cardiol 2012;10:922-929

28. Vakili BA, Okin PM, Devereux RB. Prognostic implications of left ventricular hypertrophy. Am Heart J 2001;141:334-341

29. Levy D, Garrison RJ, Savage DD et al. Prognostic implications of echocardiographically determined left ventricular mass in the Framingham heart study. NEJM 1990;322:1561-1566 